\title{
RESEARCH HIGHLIGHT OPEN Nicotinamide mononucleotide: a potential effective natural compound against insulin resistance
}

\author{
Julian Roos ${ }^{1}$, Julia Zinngrebe ${ }^{1}$ and Pamela Fischer-Posovszky (D) $^{1 凶}$ \\ Signal Transduction and Targeted Therapy (2021)6:310 \\ ; https://doi.org/10.1038/s41392-021-00723-z
}

Recently, a study published in Science by Yoshino et al. ${ }^{1}$ reported on a randomized, placebo-controlled, double-blind clinical trial to examine the effects of a 10-week nicotinamide mononucleotide (NMN) administration on human metabolism in 25 postmenopausal overweight or obese women with prediabetes. It revealed positive effects of NMN on insulin sensitivity, insulin signalling, and tissue remodelling in skeletal muscle.

Nicotinamide (NAM) and nicotinamide ribose (NR) are converted to NMN, which is the precursor of nicotinamide adenine dinucleotide $\left(\mathrm{NAD}^{+}\right)$, a co-substrate of $\mathrm{NAD}^{+}$-dependent enzymes essential for biological processes as important as redox homeostasis, gene expression, RNA processing, genomic stability, immunity and inflammation, and energy metabolism (reviewed by Xie et al. ${ }^{2}$ ). NMN is the main source of the salvage pathway to generate $\mathrm{NAD}^{+}$(Fig. 1). In addition, mammalian cells generate $\mathrm{NAD}^{+}$de novo via the Kynurenine and the Preiss-Handler pathways. ${ }^{2}$ Approximately $95 \%$ of circulating NMN is released by the liver. In rodents, NMN was reported to slow down aging and to prevent age-related disorders ranging from Alzheimer's to diabetes to metabolic dysfunction ${ }^{2}$ (see Fig. 1). This is why NMN has become the new hope in the search for longevity, the "fountain of youth". Thus, oral NMN supplementation became quite popular during the last decade, especially in the US. The study by Yoshino et al., however, is the first to deliver evidence of positive metabolic and insulin-sensitizing effects of daily NMN supplementation in humans.

Type 2 diabetes mellitus (T2DM) is one of the most serious health crises of our time with a prognosed prevalence of above $50 \%$ for 2045. Although there are 10 different US Food and Drug Administration (FDA)-approved classes of drugs for the treatment of T2DM and more than 7000 clinical trials are currently investigating new drug candidates, there is still an enormous need to develop effective medications with minor side effects. ${ }^{3}$

T2DM is a consequence of systemic insulin resistance with attenuated biological responses to normal or elevated insulin levels and subsequently impaired insulin-mediated glucose disposal. To maintain normal blood glucose levels, the pancreatic $\beta$-cells secrete more insulin resulting in compensatory hyperinsulinemia until they reach their maximal production capacity. ${ }^{3}$ Insulin resistance is caused by defects in signal transduction such as decreased insulin receptor expression and kinase activity, diminished expression and phosphorylation of insulin receptor substrate 1 (IRS-1), reduced phosphatidylinositol 3 (PI3)-kinase activity, and less glucose transporter type 4 (GLUT4) expression and translocation. ${ }^{4}$ Insulin stimulates glucose deposition and glycogen synthesis in adipose tissue, muscle and liver, and it inhibits gluconeogenesis and ketone body production in the liver. The musculature is the major site for whole-body insulin-dependent glucose uptake and therefore most relevant for glucose clearance. ${ }^{4}$

$\mathrm{NAD}^{+}$is one of the most abundant metabolites and plays an essential role as co-substrate for various enzymes. ${ }^{2}$ Among the $\mathrm{NAD}^{+}$-dependent enzymes are sirtuins, a family of class III histone deacetylases mediating post-translational modifications of again other metabolic enzymes. Thereby, $\mathrm{NAD}^{+}$availability modulates the citric acid cycle, cytosolic glycolysis, gluconeogenesis, glycogen metabolism, and mitochondrial fatty acid oxidation. ${ }^{2}$ An imbalance of $\mathrm{NAD}^{+}$homeostasis is associated with mitochondrial dysfunction, insulin resistance, and obesity. ${ }^{2}$ In line, mice on a high-fat diet showed impaired nicotinamide phosphoribosyltransferase (NAMPT)-dependent $\mathrm{NAD}^{+}$biosynthesis in metabolic organs and an adipocyte-specific Nampt knockout resulted in severe insulin resistance, which was rescued by NMN administration. Furthermore, NMN improved glucose tolerance in an ageinduced T2DM mouse model. ${ }^{2}$ The $\mathrm{NAD}^{+}$-consuming enzyme CD38 (cyclic ADP ribose hydrolase) increases with age and plays an important role in age-associated $\mathrm{NAD}^{+}$reduction. ${ }^{2} \mathrm{CD} 38$ deficiency improved glucose tolerance, especially when combined with NR supplementation. The specific CD38 inhibitor, 78c, improved age-associated glucose intolerance, muscle function, and exercise capacity. ${ }^{2}$ These and other studies highlight the essential role of $\mathrm{NAD}^{+}$and its metabolites for insulin sensitivity and glucose tolerance in rodents.

Yoshino et al. found that 10 weeks of NMN administration in humans increases muscle insulin sensitivity whereas hepatic and adipose tissue insulin sensitivity, assessed by hyperinsulinemiceuglycemic clamp, remained unaffected. ${ }^{1}$ Muscle $\mathrm{NAD}^{+}$levels of NMN-treated study participants were not altered, but NMN metabolites increased upon NMN treatment, suggesting an increased $\mathrm{NAD}^{+}$turnover (Fig. 1). It has not yet been elucidated if the elevated $\mathrm{NAD}^{+}$usage is responsible for the observed $\sim 25 \%$ increase in muscle glucose disposal. To obtain the first mechanistic insights, RNA sequencing was performed on quadriceps muscle samples after NMN supplementation. The plateletderived growth factor (PDGF) binding pathway was enriched in the treatment group suggesting its involvement in NMN-mediated insulin sensitivity (Fig. 1). The exact underlying molecular mechanism, however, remains unclear and requires further investigation.

In summary, the study by Yoshino et al. provides important first data on the safety and efficacy of NMN supplementation in humans (Fig. 1). The authors compare the NMN-induced improvement in muscle insulin sensitivity with effects induced by (i) the once famous PPARY agonist troglitazone, which was withdrawn from the

\footnotetext{
${ }^{1}$ Department of Pediatrics and Adolescent Medicine, Ulm University Medical Center, Ulm, Germany
}

Correspondence: Pamela Fischer-Posovszky (pamela.fischer@uniklinik-ulm.de)

Received: 12 June 2021 Revised: 13 July 2021 Accepted: 3 August 2021

Published online: 19 August 2021 

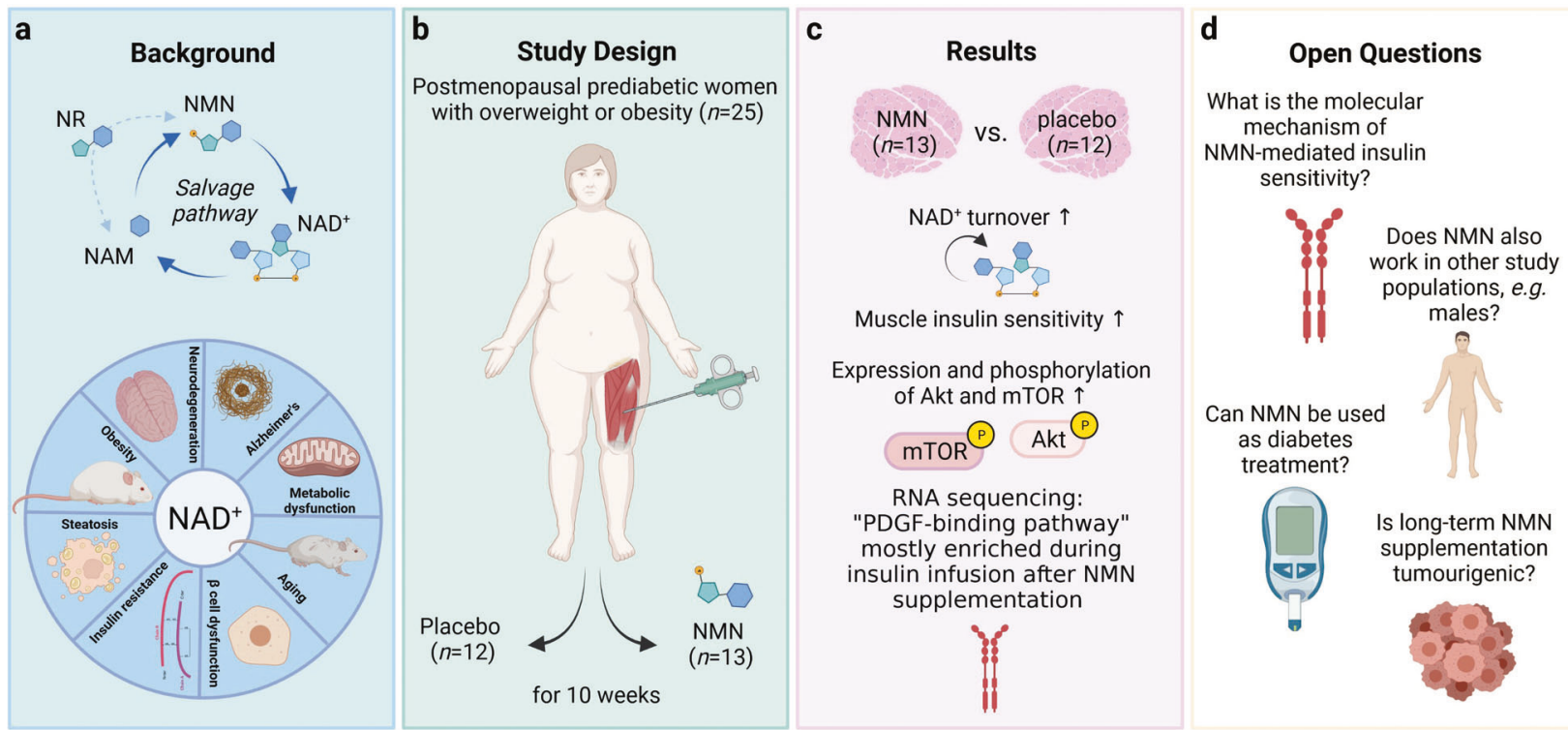

Fig. 1 a In a salvage pathway nicotinamide ribose (NR) and/or nicotinamide (NAM) are converted to nicotinamide mononucleotide (NMN), which is the precursor of nicotinamide adenine dinucleotide $\left(\mathrm{NAD}^{+}\right)$. NAD ${ }^{+}$is involved in a spectrum of diseases. b Postmenopausal prediabetic women with overweight or obesity were treated with NMN or placebo for 10 weeks and muscle biopsies revealed, $\mathbf{c}$ increased muscle $\mathrm{NAD}^{+}$turnover, insulin sensitivity, phosphorylation of mTOR and Akt, and a gene expression involving the PDGF-binding pathway in NMN-treated participants as compared to control. d Open questions, however, remain to fully assess the mechanism of NMN in mediating muscle insulin sensitivity. Furthermore, potential long-term side effects should be considered. The figure was generated with Biorender.com.

market due to safety reasons, and (ii) $10 \%$ weight loss. ${ }^{1}$ However, the study was limited to postmenopausal overweight or obese women with prediabetes and, importantly, NMN, in contrast to a significant reduction in body weight, failed to reduce circulating insulin levels or liver fat. Initial studies performed in rodents revealed positive effects of NMN on the insulin response in female mice only. ${ }^{5}$ Moreover, the use of the NAD ${ }^{+}$precursor NR, instead of NMN as supplementation, did not improve whole-body or muscle insulin sensitivity in other studies with male participants. ${ }^{1}$ Therefore, further clinical studies are required to assess whether NMN functions in a gender-specific way.

Studies in patients with T2DM will show whether NMN is able to decrease the plasma concentrations of glucose and to prevent cardiovascular and renal complications. It will be interesting to compare the effects of NMN to promising new candidates in T2DM treatment such as dual-acting and triple-acting incretin mimetics. ${ }^{3}$ Finally, the safety of long-term NMN application requires further investigation as $\mathrm{NAD}^{+}$-depleting drugs showed anti-tumour potential. ${ }^{2}$ Thus, a long-term NMN supplementation might bear the risk of driving tumour growth.

\section{ACKNOWLEDGEMENTS}

P.F.-P. holds a Heisenberg professorship (FI1700/7-1) of the German Research Foundation. J.Z. is funded by a Margarete von Wrangell scholarship (BadenWuerttemberg Ministry of Science, Research, and Arts).

\section{FUNDING}

Open Access funding enabled and organized by Projekt DEAL.

\section{ADDITIONAL INFORMATION}

Competing interests: The authors declare no competing interests.

\section{REFERENCES}

1. Yoshino, M. et al. Nicotinamide mononucleotide increases muscle insulin sensitivity in prediabetic women. Science 372, 1224-1229 (2021).

2. Xie, N. et al. $\mathrm{NAD}(+)$ metabolism: pathophysiologic mechanisms and therapeutic potential. Signal Transduct. Target Ther. 5, 227 (2020).

3. Perreault, L., Skyler, J. S. \& Rosenstock, J. Novel therapies with precision mechanisms for type 2 diabetes mellitus. Nat. Rev. Endocrinol. 17, 364-377 (2021).

4. Sylow, L., Tokarz, V. L., Richter, E. A. \& Klip, A. The many actions of insulin in skeletal muscle, the paramount tissue determining glycemia. Cell Metab. 33, 758-780 (2021).

5. Yoshino, J., Mills, K. F., Yoon, M. J. \& Imai, S. Nicotinamide mononucleotide, a key $\mathrm{NAD}(+)$ intermediate, treats the pathophysiology of diet- and age-induced diabetes in mice. Cell Metab. 14, 528-536 (2011).

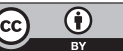

Open Access This article is licensed under a Creative Commons Attribution 4.0 International License, which permits use, sharing, adaptation, distribution and reproduction in any medium or format, as long as you give appropriate credit to the original author(s) and the source, provide a link to the Creative Commons license, and indicate if changes were made. The images or other third party material in this article are included in the article's Creative Commons license, unless indicated otherwise in a credit line to the material. If material is not included in the article's Creative Commons license and your intended use is not permitted by statutory regulation or exceeds the permitted use, you will need to obtain permission directly from the copyright holder. To view a copy of this license, visit http://creativecommons. org/licenses/by/4.0/.

(c) The Author(s) 2021 